\title{
Rapid, Simplified Technique for Heterotopic Cervical Heart Transplantation in Mice
}

\section{Shoichi lida*}

Department of Urology, Tokyo Women's Medical University, Kawada-Chyo 8-1, Shinzyuku-Ku, Tokyo, 162-8666, Japan

\begin{abstract}
Background: Methods for mouse vascularized heart transplantation using non-suture-cuff techniques have been described, but their delicate technique have prevented their widespread use. Here, we describe a simplified transplantation method with the cuff technique.

Methods: By ligating the vessels and tissues including parts of the lungs and trachea en bloc, except ascending aorta, superior and inferior vena cava, and main pulmonary artery, it was possible to reduce the time of harvesting the donor heart. In heart transplantation, using 10-0 Ethilon, it became to be easy to pass the common Carotid Artery (CA) through the cuff. And using the ascending aorta of the donor heart, it also became to be easy to cover the end of the $\mathrm{CA}$ and fix it to the arterial cuff.
\end{abstract}

Results: These modifications reduced the operating time. It typically takes 5 min to harvest the donor, 10 min to prepare the cervical vessels, and $15 \mathrm{~min}$ for anastomosis. All procedures, from donor harvest through skin closure in the recipient mice, can be completed within $40 \mathrm{~min}$, and the cold ischemic time is less than $20 \mathrm{~min}$.

Conclusions: This method can be used to investigate mechanisms of MHC-disparate heart transplantation.

Keywords: Heart transplantation; Cervical method; Cuff technique; Non-suture

\section{Protocol Text}

\section{Mice}

1. Female $\mathrm{BALB} / \mathrm{c}(\mathrm{H}-2 \mathrm{~d})$ and $\mathrm{C} 57 \mathrm{BL} / 6(\mathrm{H}-2 \mathrm{~b})$ mice were obtained from Japanese SLC (Hamamatsu, Japan). The mice were 7-12 weeks old and were housed in a specific-pathogenfree facility. Animals were used at 9-12 weeks of age. All experiments were performed in accordance with protocols approved by the Animal Care and Use Committee of the Tokyo University of Science.

2. The mice were anesthetized with $0.5 \mathrm{mg} / \mathrm{mg}$ pentobarbital, i.p. An operating microscope with $40 \times$ magnification (OMEJA; Olympus, Tokyo, Japan) was used for the procedure. The operations were performed by a single surgeon under clean, but not sterile, conditions.

\section{Preparation of the cervical cuff}

1. The cuff consisted of a 1.0-mm-long cuff body and a $1.0-\mathrm{mm}$ cuff extension, made of a long Teflon tube (outside diameter $0.6 \mathrm{~mm}$, inside diameter $0.4 \mathrm{~mm}$; Hakko, Tokyo, Japan) for the common Carotid Artery (CA).

2. A 22-gauge cuff (outside diameter $0.80 \mathrm{~mm}$ ) was used for the external jugular vein (Angiocath 2828625; Becton Dickinson, Sandy, UT).

\section{Harvesting the donor heart}

1. The following procedures should be performed to harvest the donor heart (Figure 1). The anterior thoracic cage is separated from the dorsal chest wall and is then reflected to upwards to provide maximum exposure.

2. After performing a thymectomy, $0.5 \mathrm{~mL}$ of saline solution containing $50 \mathrm{U}$ of heparin is injected into the Inferior Vena Cava (IVC) in the intrathoracic space. Next, the IVC is ligated with 6-0 silk and is divided distal to the ligatures.
3. The right Superior Vena Cava (SVC) is ligated with 6-0 silk and is divided distal to the ligatures.

4. $0.5 \mathrm{~mL}$ of cold saline solution containing $50 \mathrm{U}$ of heparin is injected into the descending aorta with a 27 -gauge needle to protect the donor myocardium.

5. The ascending aorta and Main Pulmonary Artery (MPA) are divided carefully using superfine forceps, as far distally as possible, for insertion of the recipient right $\mathrm{CA}$ and jugular vein.

6. The remaining vessels and tissues including parts of the lungs and trachea are ligated en bloc with 6-0 silk, and are divided distal to the ligatures. The donor heart is harvested. The harvested donor heart is placed in chilled Ringer's lactate solution until transplantation.

\section{Recipient preparation}

1. Under pentobarbital anesthesia, place the recipient in the supine position, with the head facing the operator. Stretch and immobilize the head with 6-0 silk holding the upper incisors to the operating board.

2. Make a midline incision in the neck from the sternum to the lower mandible.

3. Dissect free and mobilize the right external jugular vein as far as possible. Cut all of the small branches of the external jugular vein using fine-tip cautery.

*Corresponding author: Shoichi lida, Department of Urology, Tokyo Women's Medical University, Kawada-Chyo 8-1, Shinzyuku-Ku, Tokyo, 162-8666, Japan Tel: +81 33353 8111; Fax: +8135269 7401; E-mail: setogawacho@yahoo.co.jp

Received May 24, 2013; Accepted July 01, 2013; Published July 09, 2013

Citation: lida S (2013) Rapid, Simplified Technique for Heterotopic Cervical Heart Transplantation in Mice. Surgery Curr Res 3: 138. doi:10.4172/2161-1076.1000138

Copyright: (c) 2013 lida S. This is an open-access article distributed under the terms of the Creative Commons Attribution License, which permits unrestricted use, distribution, and reproduction in any medium, provided the original author and source are credited. 
4. Transect the sternomastoid muscle with fine-tip cautery to expose the right CA, which was dissected free and mobilized as far as possible.

5. Occlude the proximal portions of the CA and external jugular vein with micro-hemostat clamps; ligate the distal CA and the external portions of the external jugular vein with 6-0 silk (Figure 2 and 3 ).

6. Using fine scissors make a small hole at the bifurcation of the external jugular vein.

7. Divide the vessels between the clamps and distal ties, and irrigate the proximal cut ends with heparinized Ringer's lactate.
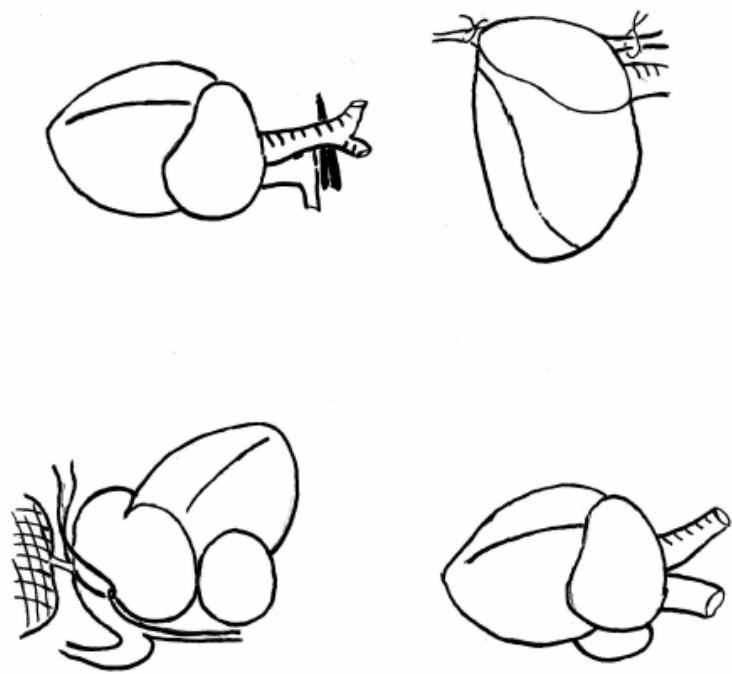

Figure 1: Donor harvest. (a) The IVC and right SVC were ligated with 6-0 silk and divided distal to the ligatures. (b) The ascending aorta and MPA were divided as far distally as possible, to insert the recipient right CA and jugular vein. (c) The remaining vessels and tissues, including parts of the lungs and trachea, were ligated en bloc with 6-0 silk and divided distal to the ligatures.
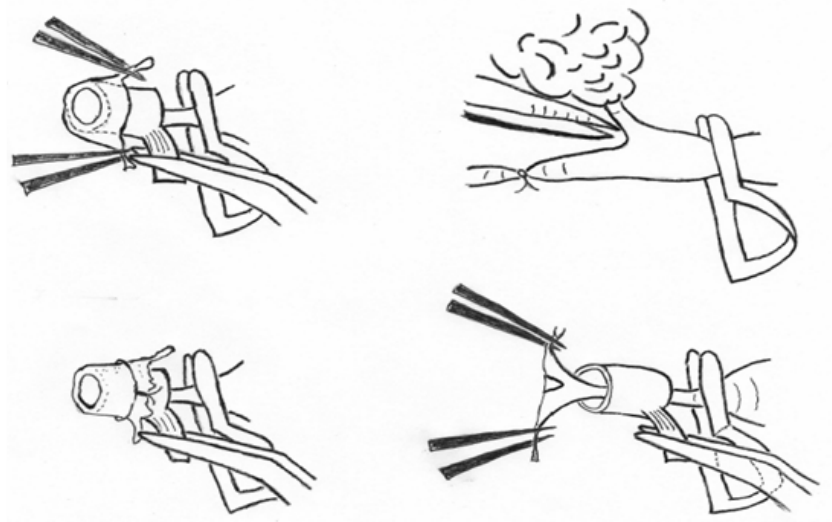

Figure 2: Preparation of the external jugular vein. The right external jugular vein was dissected free and mobilized as far as possible. All of the small branches of the external jugular vein were cut with fine-tip cautery. The proximal portion of the external jugular vein was occluded with microhemostat clamps, and the external portions of the external jugular vein were ligated with 6-0 silk. Then, a small hole was made at the bifurcation with fine scissors (a). The external vein was passed through the 22-gauge cuff with super-fine-tip forceps. On pulling the divided veins with superfine-tip forceps, the proximal end of the jugular vein was everted over the cuff $(b, c)$ and fixed to it by a circumferential ligature of 7-0 silk (d).
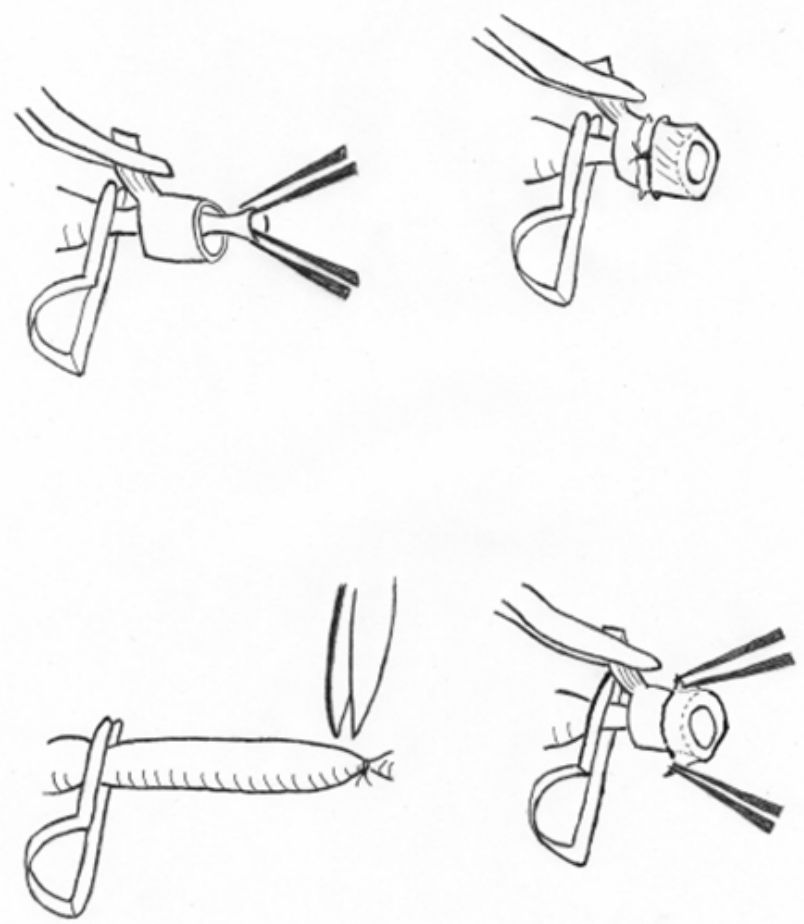

Figure 3: Preparation of the CA. The right CA was dissected free and mobilized as much as possible. The proximal portion of the CA was occluded with micro-hemostat clamps, and the distal portion of the CA was ligated with 6-0 silk (a). The CA was passed through the Teflon cuff with 10-0 Ethilon that had been placed through the edge of the artery beforehand (b). On pulling the edge of the artery with superfine-tip forceps, the proximal end of the artery was everted over the cuff (c) and fixed to it by a circumferential ligature, of 7-0 silk (d).

\section{Cervical heart transplantation}

1. To transplant the donor heart into the neck of the recipient (Figure 4), pass the CA through the cuff, using 10-0 Ethilon, placed through the edge of the artery beforehand.

2. On pulling on the edge of the artery with superfine-tip forceps (INOX5; Dumont, Switzerland), evert the proximal end of the artery over the cuff and fix it by a circumferential ligature of 7-0 silk.

3. Draw the ascending aorta of the donor heart over the end of the CA and fix it to the arterial cuff by a circular ligature of 7-0 silk.

4. Similarly, anastomose the donor MPA to the recipient external jugular vein (Figure 3). Pass the external vein through the 22-gauge cuff with superfine-tip forceps. By pulling the divided veins with superfine-tip forceps, evert the proximal end of the jugular vein over the cuff and fix in a similar fashion.

5. Anastomose the recipient jugular vein and donor MPA. By pulling on the MPA, insert the cuff fixed to the jugular vein into the MPA and fix it using a circular 7-0 silk ligature for taping (Figure 3).

6. Release the clamp on the external jugular vein first, after the anastomoses are completed, and then release the clamp on the CA. In our case, the donor-transplanted heart soon began to fibrillate, and typically reverted spontaneously to sinus rhythm within $1 \mathrm{~min}$. 


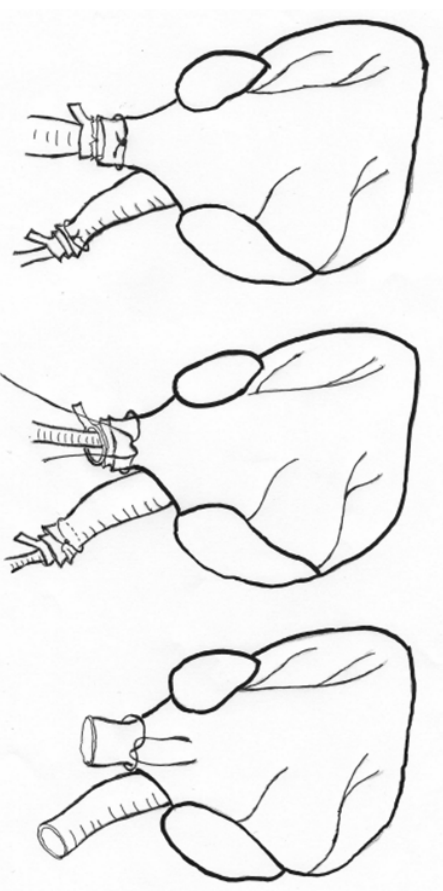

Figure 4: Anastomosis procedures. The donor heart was harvested (a). The ascending aortic artery of the donor heart was drawn over the end of the $\mathrm{CA}$ and fixed to the arterial cuff with a circular ligature of 7-0 silk. Similarly, by pulling on the MPA, a 22-gauge cuff fixed to the jugular vein was inserted into the MPA and fixed with a circular 7-0 silk ligature for taping (b and $c$ ).

\section{Representative Results}

Vascularized cervical heart transplantation from BALB/C to recipient WT B6 mice was performed. To induce donor-specific transplant tolerance, the recipient $\mathrm{C} 57 \mathrm{BL} / 6$ mice were treated with $500 \mu \mathrm{g}$ of anti-CD40L and $500 \mu \mathrm{g}$ of hCTLA4Ig (co-stimulation blockade) on the day of transplantation, and every other day for 6 days. The function of the graft was followed daily by palpating the heartbeat. Additionally, the heartbeat was visible. In total, 600 cervical heterotopic heart transplantations have been performed, with a 200 -day survival rate of $98.5 \%(394 / 400)$. The technical failure rate was less than $1 \%$.

\section{Discussion}

Chen [1] first described a suture method for cervical heterotopic heart transplantation in mice. The technical difficulties of vascularized heart transplantation in mice may be one reason why this model has never been as popular as heterotopic heart transplantation in rats.

The cuff technique has been used successfully in cervical heart transplantation in mice [2]. Tomita et al. [3] described the efficacy of the cuff technique. In this study, we modified these methods to shorten the warm ischemic time. We prepared a smaller arterial cuff (external diameter $0.6 \mathrm{~mm}$ ) and did not use any sutures. By using a smaller cuff and pulling the 10-0 Ethilon that had been placed through the edge of the artery beforehand, it became to be easy to pass the CA through the smaller cuff.

Furthermore, we chose the donor ascending aorta for arteriolar anastomosis, because the donor ascending aorta is wide and easy to pull to the cuff of the CA. For the venous anastomosis, the same direct pulling technique can also be used.

We also modified the method used to harvest the donor heart. Excluding the ascending aorta, MPA, IVC, and SVC, all of the remaining tissues were ligated en bloc. For the anastomosis, the MPA was taped with 7-0 silk and a small hole was made in the anterior wall of the MPA, following the method of Tomita et al. [3]. With these modifications, it usually took $5 \mathrm{~min}$ to harvest the donor, $10 \mathrm{~min}$ to prepare the cervical vessels, and $10 \mathrm{~min}$ to perform the anastomosis. All of the procedures, from donor harvest through skin closure in the recipient mice, can be completed in less than $40 \mathrm{~min}$, and the ischemic time was less than $20 \mathrm{~min}$.

Using this method, we have studied co-stimulation blockadeinduced heart graft acceptance and the occurrence of post-transplant chronic disease of the donor heart.

\section{Acknowledgments}

This work was aided by Sakiko Kobayashi, who prepared the antibodies, and a member of the Science Service, who took care of the experimental animals. The authors thank Dr. Hidehiro Kishimoto and Dr. Toshihiro Suzuki, Division of Immunobiology, Research Institute for Biological Sciences, Tokyo University of Science, Chiba, Japan, for their instruction and helpful discussions.

\section{References}

1. Chen $\mathrm{ZH}$ (1991) A technique of cervical heterotopic heart transplantation in mice. Transplantation 52: 1099-1101.

2. Matsuura A, Abe T, Yasuura K (1991) Simplified mouse cervical heart transplantation using a cuff technique. Transplantation 51: 896-898.

3. Tomita Y, Zhang QW, Yoshikawa M, Uchida T, Nomoto K, et al. (1997) Improved technique of heterotopic cervical heart transplantation in mice. Transplantation 64: 1598-1601 